\title{
Association of TLR3 functional variant (rs3775291) with COVID-19 susceptibility and death: a population-scale study
}

\author{
Gunanidhi Dhangadamajhi ${ }^{1}$ (D) Ronnaly Rout ${ }^{2}$
}

Received: 21 January 2021 / Accepted: 15 February 2021 / Published online: 22 February 2021

(C) Japan Human Cell Society 2021

To the Editor,

The severe acute respiratory syndrome coronavirus 2 (SARS-CoV-2) causing the recent pandemic of coronavirus disease-19 (COVID-19) has a long, positive-sense, non-segmented single-stranded RNA (ssRNA) entrapped by an envelope with spikes. Although SARS-Cov2 infection is largely asymptomatic with people of all age groups and gender are susceptible; the rate of incidence, the severity of the disease and mortality due to COVID-19 vary in different populations. The host genetic make-up has always been suspected to play an important role in almost all infectious diseases and likely to influence the COVID-19 morbidity and mortality as well. The important host genetic factors include genes involved in viral entry into host, pattern recognition receptors (PRRs) and other mediators of innate immunity [1,2]. Upon successful entry of the virus into human host, while sensing of viral infection and subsequent production of anti-viral immune response (such as production of type I and III interferons) is beneficial, deregulated inflammatory responses with cytokine storms can lead to COVID-19 immunopathogenesis and disease severity. Most notable amongst the PRRs of the innate immune systems are the toll-like receptors (TLRs) which play crucial role against coronaviruses including COVID-19. Of the 11 different TLRs, structural components of viral envelope are recognised by TLR 1,4 and 6 located on the cell surface with the strongest affinity reported for TLR 4 which is activated by oxidised

Gunanidhi Dhangadamajhi gunarmrc@gmail.com

1 Department of Biotechnology, Maharaja Sriram Chandra Bhanjadeo University (Erstwhile Known As North Orissa University), Takatpur, Baripada, Odisha 757003, India

2 Neural Developmental Biology Laboratory, Department of Life Science, National Institute of Technology, Rourkela 769008, India phospholipids produced after SARSCoV2 infection [3, 4]. On the other hand, intracellular TLRs in endosomes such as TLRs7/8 recognise single-stranded positive sense RNA whereas double-stranded RNA intermediate formed during viral replication are sensed by TLR3 $[1,3,5]$. Of these, TLR3 activation is shown to be more effective than TLR4 in mice model [5], and that the role of TLR3 activation is demonstrated to be beneficial against a wide range of RNA virus infections [2, 6, 7]. Interestingly, the high binding affinity of SARS Cov-2 non-structural protein 10 (NSP10) mRNA to TLR3 in docking study suggests a possible induction of TLR3 downstream signalling [3]. Further, protective role of TLR 3 has been documented in infections with the more closely related COVID-19 viruses such as SARS-CoV1 and the Middle East respiratory syndrome (MERS-CoV) etc. in previous studies [7, 8]. This insisted us to carry out a genetic association study to examine whether functional genetic variation in the TLR3 gene has a role in the global incidence of COVID-19 across diverse populations. Of the several mutations in TLR3, a non-synonymous mutation in exon 4 (rs3775291) has been shown to impair TLR3 expression and influence subsequent signalling cascade [9]. Further, molecular docking analysis of rs3775291 variant has revealed poor recognition of SARS-CoV-2 dsRNA compared to its wild type variant indicating a possible impaired immune protection [10]. Therefore, we hypothesised that differences in minor allele frequency of rs3775291 across different ethnic populations might have some contributary role in SARS-COV2 susceptibility and mortality.

Data on mutant allele frequency from healthy individuals were collected across different population from public-databases for genomic variants (such as 1000 Genomes Project and gnomAD) and literature searches from published articles on PubMed and Google scholars. The COVID-19 related data were acquired from worldometer site (https://www.worldometers.info/coronaviru s/) on 18th January, 2020. Data on genotype or allele 
frequencies retrieved from individual countries were subjected to Hardy Weinberg Equilibrium (HWE). Studies showing deviation from HWE were excluded from analysis. In cases where more than one data sets were obtained for a country, genotype or allele data were pooled and minor allele frequency was determined. Genetic association of TLR3 mutant (rs3775291) with COVID-19 susceptibility, mortality and percentage recovery was carried out by Pearson correlation coefficient analysis in GraphPad Prism (version 5.0) and a $P$ value $<0.05$ was considered significant. The frequency of minor allele ranged from $0.7 \%$ to $38.9 \%$ with Nigeria reporting its least prevalence and Vietnam, the highest (Supplementary Table 1). Statistical analysis revealed a significant positive correlation of TLR3 mutant (rs3775291) with SARS-Cov2 susceptibility $(P=0.0137 ; r=0.3867)$ and mortality due to Covid-19 $(P=0.0199 ; r=0.3667)$ per million of the population (Fig. 1). No correlation was observed between rs3775291 mutant and percentage recovery of COVID-19 patients. Although direct evidence on the mechanism of SARS-Cov2 incidence and higher mortality in populations harbouring the TLR3 mutant allele is not known, results of docking study predicting poor recognition of TLR3 mutant to SARS-Cov2 dsRNA [10] indicates the possibility of inadequate protective immune responses in these individuals. Moreover, TLR3 deficiency is associated with high susceptibility to RNA virus infection both in the experimental organism and clinical studies in humans [6,7]. Further, TLR3 deficiency or rs3775291 mutant allele for reduced TLR3 expression are associated with increased risk of pulmonary hypertension [11] and diabetes [12], and patients underlying these health conditions are reported to rapidly progress into Covid-19 disease severity often

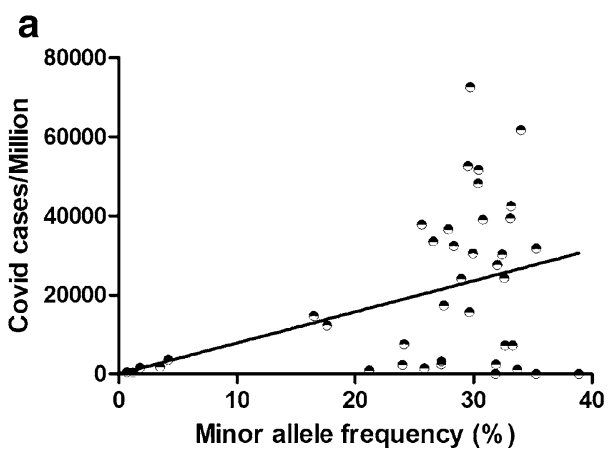

Fig. 1 Data from 40 countries were analysed. Each dot in the figure represents a country. Minor allele frequency was positively correlated with a covid-19 cases/million $(P=0.0137 ; r=0.3867)$ and b mortality/million $(P=0.0199 ; r=0.3667)$. The list of countries enrolled in the study are: Barbados, Nigeria, Gambia, Kenya, Sierra Leone, leading to death [13]. In absence of definitive evidences, we suspect that poor anti-viral immunity together with co-morbid conditions in a population with high prevalence of rs3775291 mutant allele could be the reasons for the increased susceptibility of Covid-19 infections and associated mortality. Although individuals with older age (greater than 60 years) have an increased risk of Covid-19 mortality [13], the present study includes data from overall population of all age groups and thus incompletely represents age-stratified genetic data. Further, the role of other nonsynonymous functional variant in TLR3 gene [2] or other genes which might be in linkage disequilibrium to rs3775291, and modulating Covid19 incidence and death cannot be ruled out. Besides, the risk of non-genetic factors such as pre-existing medical condition, the disparity in healthcare facility, vaccination, population mobilization and various other environmental factors are likely to affect the strength of association of the present analysis. Despite the aforesaid limitations, the finding of a significant correlation between TLR3 mutant and Covid-19 in the present investigation which retrieved data across 40 countries from 67 data sets encompassing 48835 individuals of the global population (S1) is the strength of the study. In conclusion, the TLR3 rs3775291 mutant predispose to SARS-Cov2 infection and associated mortality. A systematic analysis of disease incidence, viral load, level of anti-viral cytokines (such as IL-6, TNF, IFN, and CCL5), underlying health condition and rate of death due to Covid-19 in individuals having mutant allele compared to wild type TLR3 needs to be conducted in different race and ethnic population for better understanding and validation of the present findings.

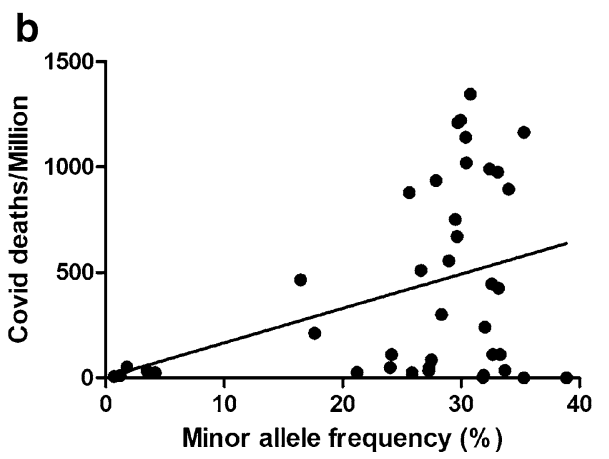

Tunisia, Morocco, USA, Colombia, Peru, Brazil, Nicaragua, China, South Korea, Taiwan, Japan, Vietnam, Finland, Scotland, Spain, Denmark, Germany, Poland, Ireland, Lithuania, Russia, Sweden, Iceland, the Netherland, Serbia, Italy, Finland, Estonia, Bulgaria, Bangladesh, India, Pakistan, Sri Lanka, Iran and Australia 
Supplementary Information The online version contains supplementary material available at https://doi.org/10.1007/s13577-021-00510 $-6$.

Funding RR is supported by DST-WOS-A grant from the Department of Science and Technology, Government of India, New Delhi.

\section{Compliance with ethical standards}

Conflict of interest We declare no competing financial interest and interpersonal-relationship influencing the work related to the present investigation.

\section{References}

1. Lee I-H, Lee J-W, Kong SW. A survey of genetic variants in SARS-CoV-2 interacting domains of ACE2, TMPRSS2 and TLR3/7/8 across populations. Infect Genet Evol. 2020;85:104507.

2. Mukherjee S, Huda S, Sinha Babu SP. Toll-like receptor polymorphism in host immune response to infectious diseases: a review. Scand J Immunol. 2019;90(1):e12771.

3. Choudhury A, Chandra Das N, Patra R, Mukherjee S. In silico analyses on the comparative sensing of SARS-CoV-2 mRNA by the intracellular TLRs of human. J Med Virol. 2020

4. Choudhury A, Mukherjee S. In silico studies on the comparative characterization of the interactions of SARS-CoV-2 spike glycoprotein with ACE-2 receptor homologs and human TLRs. J Med Virol. 2020;92(10):2105-13.

5. Zhao J, Wohlford-Lenane C, Zhao J, Fleming E, Lane TE, McCray PB, et al. Intranasal treatment with poly (I. C) protects aged mice from lethal respiratory virus infections. J Virol. 2012;86(21):11416-24.
6. Perales-Linares R, Navas-Martin S. Toll-like receptor 3 in viral pathogenesis: friend or foe? Immunology. 2013;140(2):153-67.

7. Totura AL, Whitmore A, Agnihothram S, Schäfer A, Katze MG, Heise MT, et al. Toll-like receptor 3 signaling via TRIF contributes to a protective innate immune response to severe acute respiratory syndrome coronavirus infection. MBio. 2015;6(3).

8. Biswas I, Khan GA. Coagulation disorders in COVID-19: role of toll-like receptors. J Inflamm Res. 2020;13:823.

9. Ranjith-Kumar C, Miller W, Sun J, Xiong J, Santos J, Yarbrough I, et al. Effects of single nucleotide polymorphisms on Toll-like receptor 3 activity and expression in cultured cells. J Biol Chem. 2007;282(24):17696-705.

10. Teimouri H, Maali A. Single-nucleotide polymorphisms in host pattern-recognition receptors show association with antiviral responses against SARS-CoV-2, in-silico Trial. J Med Microbiol Infect Dis. 2020;8(2):65-70.

11. Farkas D, Thompson AR, Bhagwani AR, Hultman S, Ji H, Kotha $\mathrm{N}$, et al. Toll-like receptor 3 is a therapeutic target for pulmonary hypertension. Am J Respir Crit Care Med. 2019;199(2):199-210.

12. Assmann TS, de Almeida BL, Bauer AC, Canani LH, Crispim D. Polymorphisms in the TLR3 gene are associated with risk for type 1 diabetes mellitus. Eur J Endocrinol. 2014;170(4):519-27.

13. Sanyaolu A, Okorie C, Marinkovic A, Patidar R, Younis K, Desai P, et al. Comorbidity and its Impact on Patients with COVID-19. SN Compr Clin Med. 2020;1-8.

Publisher's Note Springer Nature remains neutral with regard to jurisdictional claims in published maps and institutional affiliations. 